\title{
Nonzero Solutions of Generalized Variational Inequalities*
}

\author{
Jue Li, Yisheng Lai \\ Department of Information and Computer Science, Zhejiang Gongshang University, Hangzhou, China \\ E-mail:juelee@gmail.com \\ Received March 17, 2010; revised April 22, 2010; accepted April 29, 2010
}

\begin{abstract}
The existence of nonzero solutions for a class of generalized variational inequalities is studied by fixed point index approach for multivalued mappings infinite dimensional spaces and rflexive Banach spaces. Some new existence theorems of nonzero solutions for this class of generalized variational inequalities are established.
\end{abstract}

Keywords: Variational Inequality, Fixed Point Index of Multivalued Mappings, Nonzero Solution

\section{Introduction}

Variational inequality theory with applications are an important part of nonlinear analysis and have been applied intensively to mechanics, differential equation, cybernetics, quantitative economics, optimization theory and nonlinear programming etc. (see [1-4]).

Variational inequalities, generalized variational inequalities and generalized quasivariational inequalities were studied intensively in the last 30 years with topological method, variational method, semi-ordering method, fixed point method, minimax theorem of Ky Fan and KKM technique ([1-4]). In 1998, motivated by the paper [5], Zhu [6] studied a system of variational inequalities involving the linear operators in flexive Banach spaces by using the coincidence degree theory due to Mawhin [7]. Some existence results of positive solutions for this system of variational inequalities fhexive Banach spaces were proved.

Let $X$ be a real Banach space, $X^{*}$ its dual and $(\cdot, \cdot)$ the pair between $X^{*}$ and $X$. Suppose that $K$ is a nonempty closed convex subset of $X$.

Find $u \in K, u \neq 0$, and $w \in g(u)$ such that

$$
(A u, v-u) \geq(w, v-u), \forall v \in K
$$

where mapping $A: K \rightarrow X^{*}$ is nonlinear and $g: K \rightarrow 2^{X^{*}}$ is a multi-valued mapping.

The existence of nonzero solutions for variational inequalities is an important topic of variational inequality theory. [8] discussed the variational inequality (1) when $A$ is coercive or monotone and $g$ is set-contractive or

*This work was supported by The Zhejiang Provincial Natural Science Foundation(No.Y7080068) and the Foundation of Department of Education of Zhejiang Province(No.20070628) upper semi-continuous. [9] considered the variational inequality (1) when $A$ is single-valued continuous and $g$ is set-contractive.

On the other hand, recently, under some different conditions, $[10,11]$ obtained some existence theorems of nonzero solutions for a class of generalized variational inequalities byfixed point index approach for mu 1 ti-valued mappings in reflexive Banach space.

Based on the importance of studying the existence of nonzero solutions for variational inequalities, and motivated and inspired by recent research works in this field, in this paper, we discuss the existence of nonzero solutions for a class of generalized variational inequalities as follows:

Find $u \in K, u \neq 0$ such that

$$
\begin{aligned}
& (A u, v-u)+j(v)-j(u) \\
& \quad \geq(g(u), v-u)+(f, v-u), \forall v \in K
\end{aligned}
$$

where $A, g: K \rightarrow X^{*}$ are two nonlinear mapping and $f \in X^{*}$.

A mapping $A: X \rightarrow X^{*}$ is called hemicontinuous at $x_{0} \in X$ if for each $y \in X, A\left(x_{0}+t_{n} y\right) \underline{w}^{*} A x_{0}$ when $t_{n} \rightarrow+0$. A multivalued mapping $T: D(T) \subset X \rightarrow 2^{X^{*}}$ is said to be locally bounded in $\mathrm{v}$ if there exists a neighbourhood $V$ of $x$ for each $x \in X$ such that the set $T(V \cap D(T))$ is bounded in $X^{*}$. Suppose that $K$ is a closed convex subset of $X$ with $0 \in K$. For such $K$, the recession cone $r c K$ of $K$ is defined by $r c K=$ $\{w \in X: v+w \in K, \forall v \in K\}$. It is easily seen that the recession cone is indeed a cone and we have that $r c K \neq \varnothing$. For a proper lower semicontinuous convex functional $j: X \rightarrow R \cup\{\infty\}$ with $j(0)=0$ and $j(K) \subset$ $R_{+}=[0,+\infty)$, in the virtue of [12], the limit $\lim _{t \rightarrow+\infty} \frac{1}{t} j(t w)=$ 
$j_{\infty}(w)$ exists in $R \cup\{\infty\}$ for every $w \in X$ and $j_{\infty}$ is also a lower semicontinuous convex functional with $j_{\infty}(0)=0$ and with the property that $j(u+v) \leq$ $j(u)+j_{\infty}(v), \forall u, v \in X$.

Suppose that $K$ is a closed convex subset of $X$ and $U$ is an open subset of $X$ with $U_{K}=U \cap K \neq$ $\varnothing$. The closure and boundary of $U_{K}$ relative to $K$ are denoted by $\bar{U}_{K}$ and $\partial\left(U_{K}\right)$ respectively. Assume that $T: \bar{U}_{K} \rightarrow 2^{K}$ is an upper semicontinuous mapping with nonempty compact convex values and $T$ is also condensing, i.e., $\alpha(T(S))<\alpha(S)$ where $\alpha$ is the Kuratowski measure of noncompactness on $X$. If $x \notin T(x)$ for $x \in \partial\left(U_{K}\right)$, then the fixed point index, $i_{K}(T, U)$, is well defined(see[13]).

Proposition 1[13] Let $K$ be a nonempty closed convex subset of real Banach space $X$ and $U$ be an open subset of $X$. Suppose that $T: \bar{U}_{K} \rightarrow 2^{K}$ is an upper semicontinuous mapping with nonempty compact convex values and $x \notin T(x)$ for $x \in \partial\left(U_{K}\right)$. Then the index, $i_{K}(T, U)$, has the following properties:

1) If $i_{K}(T, U) \neq 0$, then $T$ has a fixed point;

2) For mapping $\widehat{X_{0}}$ with constant value $\left\{x_{0}\right\}$, if $x_{0} \in U_{K}$, then $i_{K}\left(\widehat{X_{0}}, U\right)=1$;

3) Let $U_{1}, U_{2}$ be two open subsets of $X$ with $U_{1} \cap U_{2}=\varnothing$.

If $x \notin T(x)$ when $x \in \partial\left(\left(U_{1}\right)_{K}\right) \cup \partial\left(\left(U_{2}\right)_{K}\right)$, then $i_{K}\left(T, U_{1} \cup U_{2}\right)=i_{K}\left(T, U_{1}\right)+i_{K}\left(T, U_{2}\right)$;

4) Let $H:[0,1] \times \bar{U}_{K} \rightarrow 2^{K}$ be an upper semicontinuous mapping with nonempty compact convex values and $\alpha(H([0,1] \times Q))<\alpha(Q)$ whenever $\alpha(Q) \neq 0, Q \subset \bar{U}_{K}$.

If $x \notin H(t, x)$ for every $t \in[0,1], x \in \partial\left(U_{K}\right)$, then $i_{K}(H(1, \cdot), U)=i_{K}(H(0, \cdot), U)$.

For every $q \in X^{*}$, let $U(q)$ be the set of solutions in $K$ of the following variational inequality

$$
\begin{aligned}
& (A u, v-u)+j(v)-j(u) \\
& \quad \geq(q, v-u)+(f, v-u), \forall v \in K
\end{aligned}
$$

Define a mapping $K_{A}: X^{*} \rightarrow 2^{K}$ by

$K_{A}(q):=U(q), \quad q \in X^{*}$.

Obviously, $K_{A}(q)=\varnothing$ if and only if the variational inequality (3) has no solution in $K$.

\section{Nonzero Solutions in $R^{n}$}

Lemma 1 Let $X$ be a separable reflexive Banach space. Suppose that $A: X \rightarrow X^{*}$ is a bounded monotone hemicontinuous mapping (i.e., for every bounded subset $D$ of $X, A(D)$ is bounded) and

$j: K \rightarrow(-\infty,+\infty]$ is a proper convex lower semicontinuous functional. Assume that there exists $v_{0} \in K s a-$ tisfying

$$
\liminf _{\|u\| \rightarrow+\infty, u \in K}\left[\left(A u, u-v_{0}\right)+j(u)-j\left(v_{0}\right)\right]>0
$$

Then for any given $f \in X^{*}$ there exists $u \in X$ such that

$$
\begin{gathered}
(A u, v-u)+j(v)-j(u) \\
\geq(f, v-u), \forall v \in X .
\end{gathered}
$$

Proof. Without loss of generality, assume that $f=0$, otherwise, set $\tilde{j}(v)=j(v)-(f, v)$. Let $K^{r}=\{x \in X$ : $\|x\| \leq r\}$. Because $X$ is a separable reflexive Banach space, for given $r$, there exists a closed convex sets sequences $K_{m}, m=1,2, \ldots$, satisfying the following conditions:

a) $K_{m} \subset K_{m+1} \subset K^{r}, m=1,2, \ldots$;

b) $K_{m} \subset X_{m}, X_{m}$ is $m$-dimensional subspace of $X$;

c) $\bigcup_{m=1}^{\infty} K_{m}$ is dense in $K^{r}$.

First, we shall verify that for each $m$, there exists $u_{m} \in K_{m}$ such that

$$
\left(A u_{m}, v-u_{m}\right)+j(v)-j\left(u_{m}\right) \geq 0, \forall v \in K_{m} .
$$

Because $X_{m}$ is a finite dimensional subspace (denoted its inner product by [.,.] ), there exists a linear continuous mapping $\pi: X^{*} \rightarrow X_{m}$ such that $(g, \omega)=$ $[\pi g, \omega]$ for all $\omega \in K_{m}$. Thus inequality (6) can be written

$$
\begin{aligned}
& [(-\pi A u+u)-u, v-u)] \\
& \quad \leq j(v)-j(u), \forall v \in K_{m} .
\end{aligned}
$$

Define a function $J_{m}(v): X_{m} \rightarrow(-\infty,+\infty]$ by

$$
J_{m}(v)=\left\{\begin{array}{c}
j(v), \quad v \in K_{m} \\
+\infty, \quad v \in X_{m} \backslash K_{m} .
\end{array}\right.
$$

Then inequality (7) can be written

$$
\begin{aligned}
& [(-\pi A u+u)-u, v-u)] \\
& \quad \leq J_{m}(v)-J_{m}(u), \forall v \in K_{m}
\end{aligned}
$$

which is equivalent to the equality

$$
u=P_{J_{m}}(-\pi A u+u)
$$

by $[2,3]$, where $P_{J_{m}}$ is an approximate mapping of $J_{m}$. 
Obviously, $\quad P_{J_{m}}(-\pi A+I): K_{m} \rightarrow K_{m}$ is continuous. According to Brouwer's fixed point theorem (see [2,3]), there exists $u_{m} \in K_{m}$ satisfying the equality (9), that is, $u_{m}$ is a solution of the variational inequality (6).

Second, we shall verify that for each $r$, there exists $u_{r} \in K^{r}$ such that

$$
\left(A u_{r}, v-u_{r}\right)+j(v)-j\left(u_{r}\right) \geq 0, \forall v \in K^{r} .
$$

In fact, $K_{m} \subset K^{r}$ and $A$ is a bounded mapping, which implies that there constant $C$ such that $\| A \mid u_{m} \leq C$ for $m=1,2, \ldots$. Since $X$ is a reflexive and $K^{r}$ is weakly closed, there exists a subsequence $\left\{u_{\mu}\right\} \subset\left\{u_{m}\right\}$ such that $u_{\mu} \stackrel{w}{\longrightarrow} u_{r}$ and $u_{r} \in K^{r}$. Because $\bigcup_{m=1}^{\infty} K_{m}$ is dense in $K^{r}$, for any given $\varepsilon>0$, there exists $u_{0} \in \bigcup_{m=1}^{\infty} K_{m}$ such that $\|u\|_{r}-u_{0} \leq \varepsilon$. It then follows from (6) that

$$
\left(A u_{\mu}, u_{\mu}-u_{0}\right) \leq j\left(u_{0}\right)-j\left(u_{\mu}\right) .
$$

when $\mu$ is sufficiently large. Thus we have

$$
\begin{aligned}
& \limsup _{\mu}\left(A u_{\mu}, u_{\mu}-u_{r}\right) \\
& \leq \limsup _{\mu}\left(A u_{\mu}, u_{\mu}-u_{0}\right)+\underset{\mu}{\limsup }\left(A u_{\mu}, u_{0}-u_{r}\right) \\
& \leq \limsup _{\mu}\left(j\left(u_{0}\right)-j\left(u_{\mu}\right)\right)+C \cdot \varepsilon .
\end{aligned}
$$

Since $j$ is a lower semicontinuous function and $\varepsilon$ is an arbitrary positive number, we have

$$
\limsup \left(A u_{\mu}, u_{\mu}-u_{r}\right) \leq 0 .
$$

This together with $A$ being a monotone hemicontinuous mapping implies that

$$
\begin{aligned}
& \liminf _{\mu}\left(A u_{\mu}, u_{\mu}-v\right) \\
& \quad \geq\left(A u_{r}, u_{r}-v\right), \forall v \in K^{r} .
\end{aligned}
$$

If $v \in \bigcup_{m=1}^{\infty} K_{m}$, it then follows from (6) that

$$
\left(A u_{\mu}, u_{\mu}-v\right) \leq j(v)-j\left(u_{\mu}\right)
$$

when $\mu$ is sufficiently large. It thus follows from (13) that

$$
\begin{aligned}
\left(A u_{r}, u_{r}-v\right) & \leq \liminf _{\mu}\left(A u_{\mu}, u_{\mu}-v\right) \\
& \leq \liminf _{\mu}\left(j(v)-j\left(u_{\mu}\right)\right) \\
& \leq j(v)-j\left(u_{r}\right), \forall v \in{ }_{m=1}^{\infty} K_{m} .
\end{aligned}
$$

Because $\bigcup_{m=1}^{\infty} K_{m}$ is dense in $K^{r}$, the above in- equality holds for all $v \in K^{r}$. therefore $u_{r}$ is a solution of the variational inequality (10).

New we shall verify that the variational inequality (5) has a solution. Taking $v=v_{0}$ in (10), we have

$$
\left(A u_{r}, u_{r}-v_{0}\right)+j\left(u_{r}\right)-j\left(v_{0}\right) \leq 0
$$

and so it then follows from condition (4) that there exists constant $C>0$ such that $\|u\|_{\text {. }} \leq C$. Taking $r>C$ then $\| u_{r}<r$ and so $u_{r}$ is an inner point of $B_{r}$. Thus for any given $\omega \in X$, we have $(1-t) u_{r}+$ $t \omega \in B_{r}$ by taking $t \in(0,1)$ small enough. Let $v=(1-t) u_{r}+t \omega$ in (10), then we obtain

$$
t\left(A u_{r}, \omega-u_{r}\right)+t\left(j(\omega)-j\left(u_{r}\right)\right) \geq 0
$$

by $j$ being a convex lower semicontinuous function. Thus

$$
\left(A u_{r}, \omega-u_{r}\right)+j(\omega)-j\left(u_{r}\right) \geq 0, \forall \omega \in X .
$$

Therefore $u_{r}$ is a solution of the variational inequality (5).

Theorem 1 Let $K$ be a nonempty unbounded closed convex set in $X=R^{n}$ with $0 \in K$. Suppose that $X \rightarrow X^{*}$ is a bounded monotone hemicontinuous mappingwith $(A u, u) \geq 0(\forall u \in K)$ and $j: K \rightarrow(-\infty,+\infty]$ is a bounded proper convex lowersemicontinuous functional with $j(0)=0$ (i.e., for every bounded subset $D$ of $K, j(D)$ is bounded). Give a continuous mapping $g: K \rightarrow X^{*}$ and $f \in X^{*}$. Assume

a) $\lim _{\|u\| \rightarrow 0} \frac{(A u, u)+j(u)}{\|u\|}=+\infty$;

b) there exists constant $\alpha \geq 0$ such that

$$
\liminf _{\|u\| \rightarrow+\infty} \frac{(A u, u)+j(u)}{\|u\|^{\alpha}\left\|^{1}\right\|}>\limsup _{\|u\| \rightarrow+\infty} \frac{\| g \mid(u)}{u^{\alpha}}(u \in K) ;
$$

c) there exists a point $u_{0} \in r c K \backslash\{0\}$ such that $\left(f, u_{0}\right) \neq 0$

Then (2) has a nonzero solution.

Proof. It is easy to see from condition (b) and Lemma 1 that the variational inequality (3) has a solution in $K$ for every $q \in X^{*}$. Define a mapping $K_{A} g: K \rightarrow 2^{K}$ by

$$
\left(K_{A} g\right)(u):=K_{A}(g(u)), u \in K
$$

Then $K_{A} g$ is an upper semi-continuous mapping with nonempty compact convex values by [10, Lemma 1]. Let $K^{R}=\{x \in K:\|x\| \leq R\}$. We shall verify that $i_{K}\left(K_{A} g, K^{R}\right)=1$ for large enough $R$ and $i_{K}\left(K_{A} g, K^{r}\right)$ $=0$ for small enough $r$. 
Firstly, define a mapping by $H:[0,1] \times \overline{K^{R}} \rightarrow 2^{K}$, $H(t, u)=t K_{A}(g(u))$. It is easily seen that $H(t, u)$ is an upper semicontinuous mapping with nonempty compact convex values. We claim that there exists large enough $R$ such that $u \notin H(t, u)$ for all $t \in(0,1), u \in \partial\left(K^{R}\right)$. Otherwise, there exist two sequences $\left\{t_{n}\right\},\left\{u_{n}\right\}, t_{n} \in[0,1]$, $t_{n} \neq 0,\|u\|_{n} \rightarrow+\infty$ such that

$$
u_{n} \in H\left(t_{n}, u_{n}\right)=t_{n} K_{A}\left(g\left(u_{n}\right)\right) \text { or } \frac{u_{n}}{t_{n}} \in K_{A}\left(g\left(u_{n}\right)\right) \text {. }
$$

Thus

$$
\begin{aligned}
& \left(A\left(\frac{u_{n}}{t_{n}}\right), v-\frac{u_{n}}{t_{n}}\right)+j(v)+j\left(\frac{u_{n}}{t_{n}}\right) \\
& \quad \geq\left(g\left(u_{n}\right), v-\frac{u_{n}}{t_{n}}\right)+\left(f, v-\frac{u_{n}}{t_{n}}\right), \forall u \in K
\end{aligned}
$$

Letting $v=0$ and denoting $z_{n}=\frac{u_{n}}{\| u_{n}}$ in (17), we obtain from (17) that

$$
\begin{gathered}
\left(\frac{t_{n}}{\|u\|_{n}}\right)^{\alpha+1}\left(A\left(\frac{u_{n}}{t_{n}}\right), \frac{u_{n}}{t_{n}}\right)+\left(\frac{t_{n}}{u_{n}}\right)^{\alpha+1} j\left(\frac{u_{n}}{t_{n}}\right) \\
\leq t_{n}^{\alpha}\left(\frac{g\left(u_{n}\right)}{\|u\|_{n}^{\alpha}}, z_{n}\right)+\left(\frac{t_{n}}{\|u\|_{n}}\right)^{\alpha}\left(f, z_{n}\right)
\end{gathered}
$$

Denote $y_{n}=\frac{u_{n}}{t_{n}} \in K$. Then $\| y_{n} \rightarrow+\infty$.We can obtain from (18) that

$$
\begin{aligned}
\frac{\left(A y_{n}, y_{n}\right)+j\left(y_{n}\right)}{\left\|y_{n}\right\|^{\alpha+1}\|\| \|} & \leq t_{n}^{\alpha} \| \frac{g\left(u_{n}\right)}{u_{n} \alpha}+\frac{\|f\|}{y_{n}^{\alpha}} \\
& \leq\left\|\frac{g\left(u_{n}\right)}{\|u\| \|}\right\|+\frac{\|f\|}{y_{n}^{\alpha}} .
\end{aligned}
$$

Hence we have

$$
\liminf _{\|u\| \rightarrow+\infty} \frac{(A u, u)+j(u)}{\|u\|^{\alpha}\left\|^{1}\right\|} \leq \limsup _{\|u\| \rightarrow+\infty} \frac{\| g t(u)}{u^{\alpha}}
$$

which contradicts to condition (b). Therefore

$$
\begin{aligned}
i_{K}\left(K_{A} g, K^{R}\right) & =i_{K}\left(H(1, \cdot), K^{R}\right) \\
& =i_{K}\left(H(0, \cdot), K^{R}\right) \\
& =i_{K}\left(\hat{0}, K^{R}\right)=1
\end{aligned}
$$

by Proposition 1(4) and (2).

Secondly, we shall verify that $i_{K}\left(K_{A} g, K^{r}\right)=0$ for small enough $r(r<1)$. In fact, there exist constants $C_{1}, C_{2}, M>0$ from the boundedness of $j$, locally boundedness of $A$ and condition (b) such that for all $u \in K^{1}$, we have

$$
\left|j\left(u+u_{0}\right)-j(u)\right| \leq C_{1},|| g \mid(u) \leq C_{2},
$$

$$
\begin{aligned}
& \left|\left(g(u), u_{0}\right)\right| \leq C_{2}\|u\| \quad \|, \not u \leq M, \\
& \left|\left(A u, u_{0}\right)\right| \leq M\|u\|
\end{aligned}
$$

Since $\left(f, u_{0}\right) \neq 0$, let $\left(f, u_{0}\right)<0$. Take $N$ large enough such that

$$
(1-N)\left(f, u_{0}\right)>C_{1}+\left(C_{2}+M\right)\left\|u_{0}\right\|
$$

Define a mapping by $H[0,1] \times \overline{K^{r}} \rightarrow 2^{K}, H(t, u)=$ $K_{A}(g(u)-t N f)$. Then $H$ is an upper semi- continuous mapping with nonempty compact convex values. We claim that there exists a small enough $r$ such that $u \notin H(t, u)$ for all $u \in \partial\left(K^{r}\right), t \in[0,1]$. Otherwise, there exist sequences $\left\{t_{n}\right\},\left\{u_{n}\right\}, t_{n} \in[0,1]$,

$u_{n} \in \partial\left(K^{r}\right), \| u_{n} \rightarrow 0$ such that $u_{n} \in H\left(t_{n}, u_{n}\right)=$ $K_{A}\left(g\left(u_{n}\right)-t_{n} N f\right)$. Thus

$$
\begin{aligned}
& \left(A u_{n}, v-u_{n}\right)+j(v)-j\left(u_{n}\right) \\
& \geq\left(g\left(u_{n}\right)-N t_{n} f, v-u_{n}\right)+\left(f, v-u_{n}\right), \forall v \in K
\end{aligned}
$$

Taking $v=0, z_{n}=\frac{u_{n}}{\| u_{n}}$, we have

$$
\begin{aligned}
& \frac{1}{\|u\|_{n} \|}\left(A u_{n}, u_{n}\right)+\frac{j\left(u_{n}\right)}{u_{n}} \\
& \quad \leq\left(g\left(u_{n}\right), z_{n}\right)+\left(1-t_{n} N\right)\left(f, z_{n}\right)
\end{aligned}
$$

Since $\frac{\left(A u_{n}, u_{n}\right)+j\left(u_{n}\right)}{\|u\|_{n}} \rightarrow+\infty$ and

$$
\begin{gathered}
\left(g\left(u_{n}\right), z_{n}\right)+\left(1-t_{n} N\right)\left(f, z_{n}\right) \\
\leq\|g(u \|)\|+(1+N) \quad f \\
\leq C_{2}+(1+N)\|f\|,
\end{gathered}
$$

we obtain a contradiction. Therefore $i_{K}\left(K_{A} g, K^{r}\right)=$ $i_{K}\left(H(0, \cdot), K^{r}\right)=i_{K}\left(H(1, \cdot), K^{r}\right)$ by Proposition 1 (4). If $i_{K}\left(H(1, \cdot), K^{r}\right) \neq 0$, then the mapping $H(1, \cdot): K \rightarrow 2^{K}$ has a fixed point $u$ in $K^{r}$ by Proposition 1(1), i.e., $u \in H(1, u)=K_{A}(g(u)-N f)$.

Thus

$$
\begin{aligned}
& (A u, v-u)+j(v)-j(u) \\
& \quad \geq(g(u)-N f, v-u)+(f, v-u), \forall v \in K
\end{aligned}
$$

Taking $v=u+u_{0}$, we have

$$
\begin{aligned}
& \left(A u, u_{0}\right)+j\left(u+u_{0}\right)-j(u) \\
& \quad \geq\left(g(u), u_{0}\right)+(1-N)\left(f, u_{0}\right)
\end{aligned}
$$

Hence

$$
\begin{aligned}
& (1-N)\left(f, u_{0}\right) \\
& \leq\left(A u, u_{0}\right)+j\left(u+u_{0}\right)-j(u)-\left(g(u), u_{0}\right) \\
& \leq M\|u\| \|+\mathbb{C}_{1}+\mathbb{C}_{2} \quad u_{0}=\left(C_{2}+M\right) \quad u_{0}+C_{1}
\end{aligned}
$$


by (21) and (23). That contradicts to (22). Therefore, $i_{K}\left(H(1, \cdot), K^{r}\right)=0$ and then $i_{K}\left(K_{A} g, K^{r}\right)=0$.

It follows from Proposition 1(3) that $i_{K}\left(K_{A} g\right.$, $\left.K^{R} \backslash K^{r}\right)=1$. Therefore there exists a fixed point $u \in K^{R} \backslash \overline{K^{r}}$ which is a nonzero solution of (2).

\section{Nonzero Solutions in Reflexive Banach Spaces}

Theorem 2 Let $X$ be a reflexive Banach space and $K \subset X$ a nonempty unbounded closed convex set with $0 \in K$. Suppose that $A: X \rightarrow X^{*}$ is a bounded monotone hemicontinuous mapping with $(A u, u) \geq 0$ for $u \in K$ and $j: K \rightarrow(-\infty,+\infty]$ is a bounded convex lower semicontinuous functional with $j(0)=0$. Assume that $g: K \rightarrow X^{*}$ is continuous from the weak topology on $X$ to the strong topology on $X^{*}$. Give $f \in X^{*}$. The following conditions are assumed to be satisfied

(a) $\left(f, u_{0}\right) \neq 0$ for some $u_{0} \in r c K \backslash\{0\}$;

(b) there constant $\alpha \geq 0$ such that $\liminf _{\|u\| \rightarrow+\infty} \frac{(A u, u)+j(u)}{\|u\|^{\alpha}\left\|^{1}\right\|}>\limsup _{\|u\| \rightarrow+\infty} \frac{\|g\|(u)}{u^{\alpha}}(u \in K) ;$

(c) $\liminf _{\substack{w \\ u_{s} \rightarrow 0}} j\left(u_{s}\right)>0$.

Then (2) has a nonzero solution.

Proof. It is easily seen that $\lim _{\|u\| \rightarrow 0} \frac{A(u, u)+j(u)}{\|u\|}=$ $+\infty$ by the condition (c). Let $F \subset X$ be a finite dimensional subspace containing $u_{0}$. We shall show that all conditions in Theorem 1 are satisfied on space $F$.

Denote $K_{F}=K \cap F$ which is a nonempty unbounded closed convex set. Let $j_{F}: F \rightarrow X$ be an injective mapping and $j_{F}^{*}: X^{*} \rightarrow F^{*}$ its dual mapping. Denote $A_{F}=j_{F}^{*}(A \mid F): F \rightarrow F^{*}, g_{F}=j_{F}^{*}\left(g \mid K_{F}\right): K_{F}$ $\rightarrow F^{*}$. We know that $A_{F}=j_{F}^{*} A j_{F}, g_{F}=j_{F}^{*} g j_{F}$. Then, $A_{F}, g_{F}$ are hemicontinuous and continuous respectively.

For $x_{1}, x_{2} \in K_{F}$, we have

$$
\begin{aligned}
& \left(A_{F}\left(x_{1}\right)-A_{F}\left(x_{2}\right), x_{1}-x_{2}\right) \\
& =\left(j_{F}^{*} A\left(x_{1}\right)-j_{F}^{*} A\left(x_{2}\right), x_{1}-x_{2}\right) \\
& =\left(A x_{1}-A x_{2}, j_{F}\left(x_{1}-x_{2}\right)\right) \\
& =\left(A x_{1}-A x_{2}, x_{1}-x_{2}\right) \geq 0
\end{aligned}
$$

by the monotony of $A$. This means that $A_{F}$ is monotone. On the other hand, $j_{F}^{*} f \in F^{*}$ and $\left(j_{F}^{*} f, u_{0}\right)=$ $\left(f, j_{F} u_{0}\right)=\left(f, u_{0}\right) \neq 0$. Similarly, we have

$$
\begin{array}{r}
\liminf _{\|u\| \rightarrow+\infty} \frac{\left(A_{F} u, u\right)+j(u)}{\|u\|^{\alpha}\left\|^{1}\right\|}>\limsup _{\|u\| \rightarrow+\infty} \frac{\|g\|_{F}(u)}{u^{\alpha}} \\
\left(u \in K_{F}\right) .
\end{array}
$$

Therefore all conditions in Theorem 1 are satisfied on space $F$ and so there exists $u_{F} \in K_{F}, u_{F} \neq 0$ such that

$$
\begin{aligned}
& \left(A_{F}\left(u_{F}\right), v-u_{F}\right)+j(v)-j\left(u_{F}\right) \\
& \quad \geq\left(g_{F}\left(u_{F}\right), v-u_{F}\right)+\left(j_{F}^{*} f, v-u_{F}\right), \forall v \in K_{F}
\end{aligned}
$$

It yields that

$$
\begin{aligned}
& \left(A\left(u_{F}\right), v-u_{F}\right)+j(v)-j\left(u_{F}\right) \\
& \quad \geq\left(g\left(u_{F}\right), v-u_{F}\right)+\left(f, v-u_{F}\right), \forall v \in K_{F}
\end{aligned}
$$

Taking $v=0$, we get

$$
\begin{array}{r}
\left(A u_{F}, u_{F}\right)+j\left(u_{F}\right) \leq\left(g\left(u_{F}\right), u_{F}\right)+\left(f, u_{F}\right) . \text { Hence } \\
\frac{\left(A u_{F}, u_{F}\right)+j\left(u_{F}\right)}{\|u\|\left\|^{\alpha+1}\right\| \|} \leq \frac{\| g \mid\left(u_{F}\right)}{u_{F}^{\alpha}}+\frac{\|f\|}{u_{F}^{\alpha} .}
\end{array}
$$

This together with condition (b) implies that there exists a constant $M>0$ such that $\| u_{F} \leq M$ for all finite dimensional subspace $F$ containing $u_{0}$. Since $X$ is reflexive and $K$ is weakly closed, with a similar argument to that in the proof of Theorem 2 in [10] (also see [8]), we shall show that there exists $u^{\prime} \in K$ such that for every finite dimensional subspace $F$ containing $u_{0}, u^{\prime}$ is in the weak closure of the set $V_{F}=\bigcup_{F \subset F_{1}}\left\{u_{F_{1}}\right\}$ where $F_{1}$ is a finite dimensional subspace in $X$.

In fact, since $V_{F}$ is bounded, we know that $\overline{\left(V_{F}\right)^{w}}$

(the weak closure of the set $V_{F}$ ) is weakly compact. On the other hand, let $F^{1}, F^{2}, \cdots, F^{m}$ be finite dimensional subspaces containing $u_{0}$. Define $F^{(m)}:=$ $\operatorname{span}\left\{F^{1}, F^{2}, \cdots, F^{m}\right\}$. Then $F^{(m)}$ containing $u_{0}$ is a finite dimensional subspace. Hence, $\bigcap_{i=1}^{m} V_{F^{i}}=$

$\bigcap_{i=1}^{m}\left(\bigcup_{F^{i} \subset F_{1}}\left\{u_{F_{1}}\right\}\right)=\bigcup_{F^{(m)} \subset F_{1}}\left\{u_{F_{1}}\right\} \neq \varnothing$, then $\bigcap_{F} \overline{\left(V_{F}\right)^{w}} \neq$ $\varnothing$. That is to say, there exists $u^{\prime} \in K$ such that for every finite dimensional subspace $F$ containing $u_{0}$, $u^{\prime}$ is in the weak closure of the set $V_{F}=\cup_{F \subset F_{1}}\left\{u_{F_{1}}\right\}$.

Now let $v \in K$ and $F^{\prime}$ a finite dimensional subspace of $X$ which contains $u_{0}$ and $v$. Since $u^{\prime}$ belongs to 
the weak closure of the set $V_{F^{\prime}}=\bigcup_{F^{\prime} \subset F_{1}}\left\{u_{F_{1}}\right\}$. We may find a sequence $\left\{u_{F_{\alpha}}\right\}$ in $V_{F^{\prime}}$ such that $u_{F_{\alpha}} \stackrel{w}{\rightarrow} u^{\prime}$. However, $u_{F_{\alpha}}$ satisfies the following inequality

$$
\begin{aligned}
& \left(A u_{F_{\alpha}}, v-u_{F_{\alpha}}\right)+j(v)-j\left(u_{F_{\alpha}}\right) \\
& \quad \geq\left(g\left(u_{F_{\alpha}}\right), v-u_{F_{\alpha}}\right)+\left(f, v-u_{F_{\alpha}}\right)
\end{aligned}
$$

The monotony of $A$ implies that

$$
\begin{aligned}
& \left(A v, v-u_{F_{\alpha}}\right)+j(v)-j\left(u_{F_{\alpha}}\right) \\
& \quad \geq\left(g\left(u_{F_{\alpha}}\right), v-u_{F_{\alpha}}\right)+\left(f, v-u_{F_{\alpha}}\right)
\end{aligned}
$$

Letting $u_{F_{\alpha}} \stackrel{w}{\rightarrow} u^{\prime}$ yields that

$$
\begin{aligned}
& \left(A v, v-u^{\prime}\right)+j(v)-j\left(u^{\prime}\right) \\
& \quad \geq\left(g\left(u^{\prime}\right), v-u^{\prime}\right)+\left(f, v-u^{\prime}\right), \forall v \in K
\end{aligned}
$$

Thus

$$
\begin{aligned}
& \left(A u^{\prime}, v-u^{\prime}\right)+j(v)-j\left(u^{\prime}\right) \\
& \quad \geq\left(g\left(u^{\prime}\right), v-u^{\prime}\right)+\left(f, v-u^{\prime}\right), \forall v \in K
\end{aligned}
$$

by Minty's Theorem [2,3]. We claim that $u^{\prime} \neq 0$. Otherwise, $u_{F_{\alpha}} \stackrel{w}{\rightarrow} 0$. Taking $v=0$ in (24) yields that

$$
\begin{aligned}
j\left(u_{F_{\alpha}}\right) & \leq-\left(A u_{F_{\alpha}}, u_{F_{\alpha}}\right)+\left(g\left(u_{F_{\alpha}}\right), u_{F_{\alpha}}\right)+\left(f, u_{F_{\alpha}}\right) \\
& \leq\left(g\left(u_{F_{\alpha}}\right), u_{F_{\alpha}}\right)+\left(f, u_{F_{\alpha}}\right)
\end{aligned}
$$

The right side of the above inequality tends to 0 , which contradicts to the condition (c). Therefore $u^{\prime}$ is a nonzero solution of (2).

\section{References}

[1] G. X. Z. Yuan, "KKM Theory and Applications in Nonlinear Analysis," Marcel Dekker, New York, 1999.

[2] D. Kinderlehrer and G. Stampacchia, "An Introduction to Variational Inequalities and Their Applications," Academic Press, New York, 1980.

[3] S. S. Chang, "Variational Inequality and Complementarity Problem Theory with Applications," Shanghai Scientific Technology and Literature Press, Shanghai, 1991.

[4] F. Facchinei and J. S. Pang, "Finite-dimensional Variational Inequality and Complementarity Problems," SpringerVerlag, New York, 2003.
[5] A. Szulkin, "Positive Solutions of Variational Inequalities: A Degree Theoretic Approch," Journal of Differential Equations, Vol. 57, No. 1, 1985, pp. 90-111.

[6] Y.G. Zhu, "Positive Solutions to A System of Variational Inequalities," Applied Mathematics Letters, Vol. 11, No. 4, 1998, pp. 63-70.

[7] J. Mawhin, "Equivalence Theorems for Nonlinear Operator Equations and Coincidence Degree Theory for Some Mappings in Locally Convex Topological Vector Spaces," Journal of Differential Equations, Vol. 12, 1972, pp. 610-636.

[8] Y. Lai, "Existence of Nonzero Solutions for A Class of Generalized Variational Inequalities," Positivity, Vol. 12, No. 4, 2008, pp. 667-676.

[9] K. Q. Wu and N. J. Huang, "Non-Zero Solutions for A Class of Generalized Variational Inequalities in Reflexive Banach Spaces," Applied Mathematics Letters, Vol. 20, No. 2, 2007, pp. 148-153.

[10] Y. Lai and Y. G. Zhu, "Existence Theorems for Solutions of Variational Inequalities," Acta Mathematica Hungarica, Vol. 108, No. 1-2, 2005, pp. 95-103.

[11] J. H Fan and W. H. Wei, "Nonzero Solutions for A Class of Set-Valued Variational Inequalities in Reflexive Banach Spaces," Computers Mathematics with Applications, Vol. 56, No. 1, 2008, pp. 233-241.

[12] D. D. Ang, K. Schmitt and L. K. Vy, "Noncoercive Variational Inequalities: Some Applications," Nonlinear Analysis: Theory, Methods \& Applications, Vol. 15, No. 6, 1990, pp. 497-512.

[13] P. M. Fitzpatrick and W. V. Petryshyn, "Fixed Point Theorems and The Fixed Point Index for Multivalued Mappings in Cones," Journal of the London Mathematical Society, Vol. 12, No. 2, 1975, pp. 75-85.

[14] M. S. R. Chowdhury and K. K. Tan, "Generalization of Ky Fan's Minimax Inequality with Applications to Generalized Variational Inequalities for Pseudo-Monotone Operators and Fixed point theorems," Journal of Mathematical Analysis and Applications, Vol. 204, No. 3, 1996, pp. 910-929.

[15] K. Deimling, "Nonlinear Functional Applications," SpringerVerlag, New York, 1985.

[16] D. Pascali and S. Sburlan, "Nonlinear Mappings of Monotone Type," Sijthoff \& Noordhoff International Publishers, Bucuresti, 1976.

[17] W. V. Petryshyn, "Multiple Positive Fxed Points of Multivalued Condensing Mappings with Some Applications," Journal of Mathematical Analysis and Applications, Vol. 124, 1987, pp. 237-253. 